\title{
Identification of women at high genetic risk of breast cancer through the National Health Service Breast Screening Programme (NHSBSP)
}

Deepthi de Silva, Fiona Gilbert, Gillian Needham, Heather Deans, Peter Turnpenny, Neva Haites

\begin{abstract}
Breast cancer is a multifactorial disease with an inherited predisposition being implicated in around $5 \%$ of all cases. Using previous epidemiological data assessing risks for the relatives of women with breast cancer, we have identified 154 women (from a screened population of 35 505) and 289 of their relatives between 50 and 64 years who have more than twice the age related risk of developing breast cancer. This constitutes $1 \cdot 24 \%$ of the breast screening population attending the North East Scotland NHSBSP. For each woman identified to be at high risk, we have found 1.87 female relatives between 50 and 64 years and 1.85 relatives under 50 years also to be at high risk. Around $78 \%$ of the women identified with a significant family history of breast or other cancer have attended for counselling about their risks. The breast screening programme can be used to identify women at high risk of breast cancer in order to offer them (and their relatives) access to genetic counselling and appropriate screening.
\end{abstract}

( $\mathcal{F}$ Med Genet 1995;32:862-866)

The North East Scotland Breast Screening Centre covers a large geographical area including the Grampian region and the Orkney and Shetland islands. The population covered comprises approximately 42300 women between the ages of 50 and 64 years. ${ }^{12}$

Breast cancer is a multifactorial disease with a hereditary predisposition being a major risk factor in around $5 \%$ of women with this disease..$^{3-5}$ The relatives of women with breast cancer are at increased risk, especially when the index case is premenopausal at the time of diagnosis, has bilateral breast or other tumours (especially ovarian cancer), or when more than one first degree relative is affected in the family. ${ }^{67}$ This increased breast cancer risk is well recognised for the premenopausal, first degree relatives of women who are diagnosed under the age of 40 years. ${ }^{67}$ The risks to the first degree relatives of older women (over 40 years) is less well recognised although some women in this group remain at increased risk because of an inherited predisposition. Mettlin et $a l,{ }^{8}$ in a case control study of 779 breast cancer patients and 1558 age matched controls, showed that the relative risk (RR) of breast cancer increases for women over 55 years with a positive family history. The $R R$ is 1.8 if only one relative is affected, 3.3 if more than one first degree relative is affected, and 2.3 if bilateral disease is present. In addition, in the families with a dominantly inherited predisposition to breast cancer, heterogeneity in the age of onset of cancer has been observed and women over the age of 50 from these families remain at increased risk compared with the general population. ${ }^{9-11}$

Breast screening was introduced in the UK in 1986 with women between 50 and 64 invited every three years for either one or two view mammography. ${ }^{1}$ Randomised controlled trials have shown that screening can reduce mortality by up to $30 \%$ in this age group. ${ }^{1}$ It is recognised that the total numbers of cancers diagnosed can be increased if the frequency of screening is less than three years, although the cost per case will be proportionally higher. ${ }^{1}$ Most European countries with population screening have introduced two yearly screening programmes. Targeting more frequent screening to women identified to be at high risk because of a hereditary predisposition is a rational method of using the available resources, although it is accepted that no trial has shown the mortality benefit of this approach.

Here we present the results of a descriptive, pragmatic study to identify the number of women in the breast screening population at greater than twice the age related risk of developing breast cancer and assessment of the uptake of genetic counselling by those women considered to be at increased risk.

\section{Methods}

Women attending for breast screening were asked by the radiographer if they had a family history of breast cancer and the answers were recorded. The radiologist reviewed the information and those women with a family history fulfilling the following criteria were recalled to the breast screening centre by letter, which explained that their mammogram was normal and that the reason for recall was their family history.

The criteria used were the following:

(1) First degree relative with bilateral breast cancer. 
NHSBSP Study

Results

October 1990-September 1993

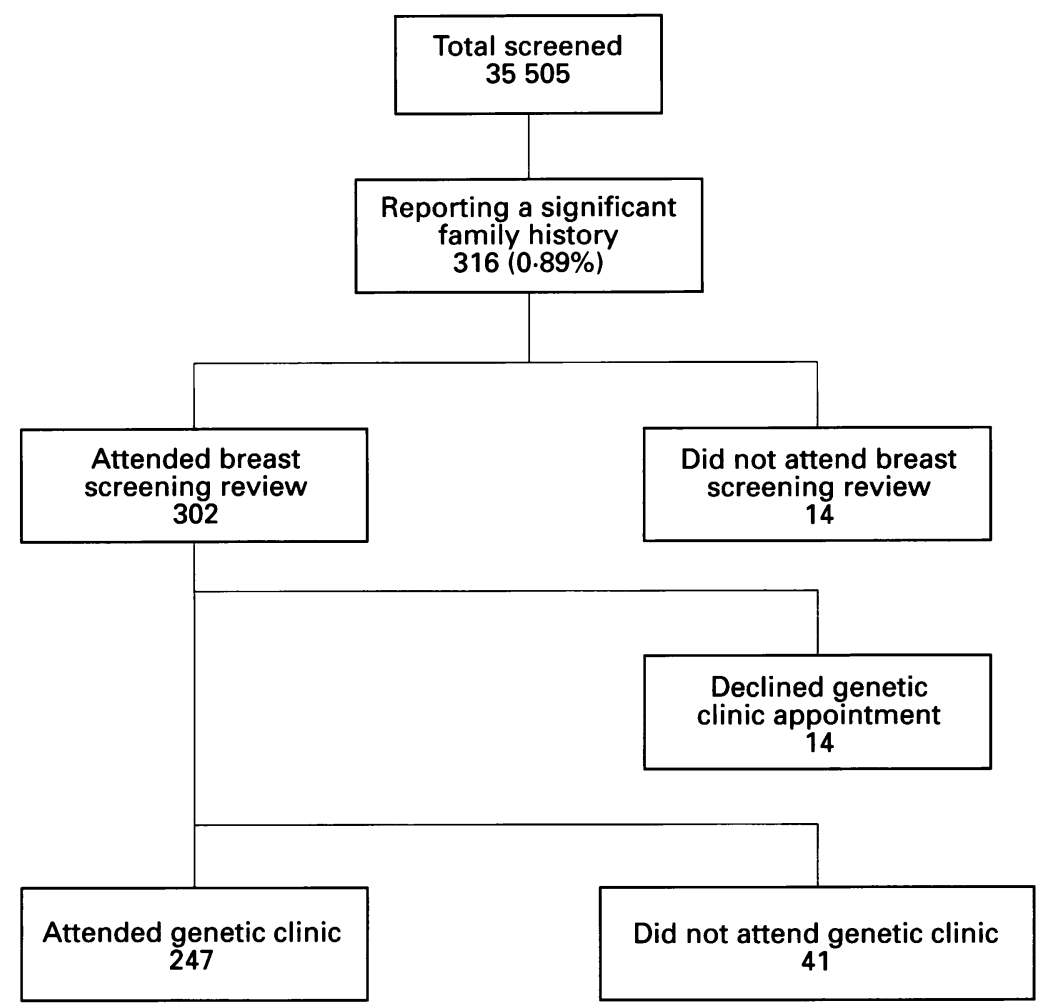

NHSBSP study results, October 1990 to September 1993

Table 1 Women identified by the radiologists and their referral categories

\begin{tabular}{lcc}
\hline & No referred & Attended \\
\hline (1) 1st degree rel with bilat breast cancer & 27 & $26(96 \%)$ \\
(2) Two 1st degree rels with breast cancer & 66 & $61(92 \%)$ \\
(3) 1st degree rel with breast cancer <40 & 115 & $92(80 \%)$ \\
(4) Male rel with breast cancer & 2 & $2(100 \%)$ \\
(5) Dominant family history & 78 & $66(85 \%)$ \\
Total & 288 & $247(86 \%)$ \\
\hline
\end{tabular}

(2) Two first degree relatives with breast cancer (including breast and ovarian cancer).

(3) First degree relative with breast cancer under 40 years.

(4) First or second degree male relative with breast cancer.

(5) An apparently dominant family history of breast cancer (three or more affected relatives in at least two generations).

At the second attendance, within two weeks of the screening mammogram, the radiologist reviewed the family history and performed a clinical examination of the breasts. A second view (cranio-caudal) mammogram was also performed. The women with a family history fulfilling the above criteria were also offered an opportunity to attend the genetic clinic for counselling (about their family history and its significance to themselves and other family members) and informed that if their risks were confirmed to be significantly increased, they would be offered more frequent screening at 18 monthly intervals.
At the genetic clinic the family history details were obtained and later verified if possible by review of hospital case records, cancer register information or death certificates. Risk assessment was performed using the risk calculations from the $\mathrm{CASH}^{12}$ (cancer and steroid hormone) study and Houlston et $a l^{7}$ for the families with a predominantly premenopausal age of onset of breast cancer. The study data of Mettlin $e t a l^{8}$ were used for estimating risk for women reporting two first degree relatives with postmenopausal breast cancer, as the CASH study is restricted to probands affected under the age of 55 and Houlston et $^{a l^{7}}$ do not provide risk estimates for the families with two affected first degree relatives. The counselling involved discussing the woman's individual risk compared with the population risk of breast cancer, the availability of mammography, and the benefits (including the detection of presymptomatic breast cancers) and disadvantages (radiation risks, insensitivity of mammography) of this. Other at risk or affected family members were identified and contacted where appropriate by the consultand. In families with a possible dominantly inherited risk, the mode of inheritance of the predisposition was discussed. In the families with a predisposition to ovarian cancer, screening and prophylactic surgery was also discussed.

\section{Results}

In the three year period from October 1990 to September 1993, 35505 women were screened at the North East Scotland breast screening centre, representing a $80.9 \%$ uptake for breast screening. ${ }^{13}$ Of the screened population, 316 $(0 \cdot 89 \%)$ women were considered by the radiologists to fulfil the criteria for being at increased risk (figure). Fourteen women did not attend the radiology review appointment and 14 women declined the offer to be counselled at the genetic clinic.

A further 41 women did not attend the genetic clinic appointment. One had reported a first degree relative with bilateral breast cancer, five had reported having two or more first degree relatives with breast cancer, 23 had a first degree relative under 40 with breast cancer, and 12 were referred because of a family history suspicious of a dominantly inherited predisposition to cancer.

Of the women identified by the radiologists to be at increased risk, $247(78 \%)$ attended the genetic clinic (table 1). Of these, 26 women reported a first degree relative with bilateral breast cancer, 61 women reported two first degree relatives with breast cancer, 92 women reported a first degree relative under 40 years with breast cancer, two reported a male relative with breast cancer, and 66 women reported a family history suspicious of a dominantly inherited predisposition to cancer.

Following risk assessment, 93 (37\%) women were found to be at less than twice the age related risk of breast cancer. Seventy-four of these women had a first degree relative under 40 years with breast cancer; the remaining women's family history was refuted following 
Table 2 Risk assessment following visit to the genetics clinic

\begin{tabular}{ll}
\hline Less than twice the population age related risk & $93(38 \%)$ \\
Greater than twice the population age related risk & $91(37 \%)$ \\
Autosomal dominant families & $63(26 \%)$ \\
Total & 247
\end{tabular}

review of the family history information (tables 2 and 3 ).

A total of $91(36 \%)$ women were found to be at greater than twice the age related risk of breast cancer but were not from families with an apparently dominantly inherited breast cancer predisposition. This included 22 women with a family history of bilateral breast cancer in a first degree relative, 39 with two first degree relatives affected with breast cancer, 11 with a first degree relative under 40 , and one with a male relative with breast cancer. Eighteen had initially reported a family history consistent with a dominantly inherited cancer predisposition but their overall risk was modified by additional family history information, hormonal risk factors, and review of the family history from hospital records and cancer register information (tables 2 and 3).

Sixty-three $(26 \%)$ of the women seen at the genetic clinic were from families where there was an apparently dominantly inherited risk for breast and other cancers with three or more relatives affected over at least two generations. Thirty-nine of these women had reported a family history suspicious of a dominantly inherited predisposition and this was confirmed following verification of the diagnoses after attendance at the genetic clinic. Additional family history information was also identified to categorise the remaining 14 women into families with a possible dominantly inherited cancer predisposition. The referral criteria for these women included three with a first degree relative with bilateral breast cancer, 14 with two first degree relatives with breast cancer, and seven with a first degree relative under 40. Among these families where autosomal dominant inheritance was likely, 20 reported a predominantly postmenopausal onset of breast cancer, 21 were perimenopausal in onset, and 22 were premenopausal. Many women in this latter category have "lived through" much of their prior risk, and they were counselled accordingly. Of the women in dominant families, 13 reported breast and ovarian cancer and three had breast, ovarian, and gastrointestinal (GI)

Table 3 Risk assessment from referral categories

\begin{tabular}{|c|c|c|c|c|}
\hline \multirow[t]{2}{*}{ Referral category } & \multicolumn{3}{|c|}{ Assessed risk after genetic clinic } & \multirow[t]{2}{*}{ Total } \\
\hline & $\begin{array}{l}<\times 2 \text { age } \\
\text { risk } \\
\text { (\% of total }\end{array}$ & $\begin{array}{l}>\times 2 \text { age } \\
\text { risk } \\
\text { from referra }\end{array}$ & $\begin{array}{l}A D^{*} \\
\text { category) }\end{array}$ & \\
\hline $\begin{array}{l}\text { 1st degree rel with bilat breast cancer } \\
\text { Two 1st degree rels with breast cancer } \\
1 \text { st degree rel with breast cancer }<40 \\
\text { Male rel with breast cancer } \\
\text { Dominant family history }\end{array}$ & $\begin{aligned} & 1(3) \\
& 8(13) \\
& 74(80) \\
& 1(50) \\
& 9(14)\end{aligned}$ & $\begin{array}{l}22(85) \\
39(64) \\
11(12) \\
1(50) \\
18(27)\end{array}$ & $\begin{array}{r}3(12) \\
14(23) \\
7 \quad(8) \\
0 \\
39(59)\end{array}$ & $\begin{array}{r}26 \\
61 \\
92 \\
2 \\
66\end{array}$ \\
\hline Total & 93 & 91 & 63 & 247 \\
\hline
\end{tabular}

* $\mathrm{AD}=$ autosomal dominant families
Table 4 At risk relatives identified following genetic clinic visit

\begin{tabular}{lcc}
\hline Assessed risk category & Under 50 years & 50-64 years \\
\hline $\begin{array}{l}<\times 2 \text { age risk } \\
(\mathrm{n}=93)\end{array}$ & 58 & 0 \\
$\begin{array}{l}>\times 2 \text { age risk } \\
(\mathrm{n}=91)\end{array}$ & 107 & 153 \\
$\begin{array}{l}\text { Autosomal dominant } \\
\text { families } \\
(\mathrm{n}=62)\end{array}$ & 121 & 136 \\
Total & 286 & \\
\hline
\end{tabular}

cancer in their family history. Other cancer family histories included eight who reported GI and breast cancer, three with breast, GI and endometrial cancer, two with breast, GI, and central nervous system tumours, and two with breast cancer and leukaemia/lymphoma. In the families with breast, colorectal, ovarian, and uterine cancer, screening for these tumours has also been offered.

Using available pedigree information, we have also identified 289 women between 50 and 64 and 286 women under 50 years also at increased risk of breast cancer (table 4 ). In the families with a probable dominantly inherited predisposition, 136 women over 50 and 121 at risk women under this age were identified. In the women at intermediate risk, 153 at risk relatives between 50 and 64 and 107 under 50 were identified. In the group whose risk was less than twice the lifetime risk, 58 relatives under 50 years at greater than twice their age related risks were identified. A total of 442 women at greater than twice the age related risk of breast cancer have been identified in this breast screening population including the 153 consultands and their at risk relatives between the ages of 50 and 64 . This represents $1 \cdot 24 \%$ of the North East Scotland NHSBSP population.

These results represent kindreds identified through the NHSBSP. Women who were members of a family identified through this study were counted as at risk family members.

Four women in this study have developed cancer. Two of them are from families with breast and ovarian cancer. The first woman, who reported a sister with breast cancer aged 28 and a mother with ovarian cancer aged 71 , presented aged 54 with a grade 3 breast cancer at her 18 monthly mammogram. In addition to a mastectomy, she had bilateral prophylactic oophorectomy performed in view of her family history and following discussion with the relevant clinicians. Although the ovaries were macroscopically normal, histological examination showed that she had bilateral ovarian adenocarcinoma. Following surgery, she also received adjuvant radiotherapy, chemotherapy, and Tamoxifen. Six months postoperatively, she presented with ascites and died suddenly from a pulmonary embolus. No recurrence of the ovarian cancer was demonstrable in her abdomen at necropsy.

The second woman reported a family history of breast cancer in a sister and mother aged 30 and 28 respectively and ovarian cancer in her maternal grandmother in her late $50 \mathrm{~s}$. She presented for her second screening mam- 
mogram at an 18 monthly interval with a palpable right breast tumour histologically proven to be a grade 2 invasive ductal carcinoma with no node involvement. Her mammogram also showed bilateral breast microcalcification. Following further discussion, she requested a prophylactic bilateral mastectomy; histological examination of the right breast showed a second focus of papillary intraduct carcinoma of large cell type and the left breast had solid type ductal carcinoma in situ with one small focus of Paget's disease in the nipple. She had previously undergone a prophylactic oophorectomy. She remains well two years later.

The third subject reported a history of breast and renal carcinoma in her mother, colon cancer in four maternal uncles, and ovarian carcinoma in her niece aged 35. She presented aged 63 with disseminated intra-abdominal carcinoma with a probable primary in her stomach. In addition, she had an ovarian mass which may have been metastatic or a primary tumour, but it was not investigated further. She died aged 63 from disseminated carcinomatosis.

The fourth woman reported a family history of two sisters aged 37 and 45 with unilateral breast carcinoma. She presented at the age of 64 with a unilateral, invasive ductal carcinoma at screening mammography.

\section{Discussion}

This is the first attempt (in the UK) at a population based study to assess the incidence of familial breast cancer and offer genetic counselling and more frequent mammography for those at greater than twice the age related risk of breast cancer. It shows that a small but significant number of women in the breast screening population between 50 and 64 years report a family history suspicious of an inherited predisposition to cancer and which significantly increases the risk of breast cancer to them or their family members.

Identifying high risk women at attendance at the NHSBSP has several advantages. Most cancer family clinics offer their services to the women who seek their help but are unable to offer equity of access for genetic counselling and screening for other families at risk. As older women with better information about the family history are approached, more extensive pedigree information becomes available, making risk assessment easier. In the families with a high risk of premenopausal breast cancer, the first contact is with the women who have a low remaining lifetime risk and who are able to discuss the issue with the younger family members. As the mortality benefits of mammography in the 50 to 65 age group have been shown, the targeting of more frequent screening to the high risk women in the NHSBSP is a rational use of resources.

The major disadvantage of this approach is the risk of causing increased anxiety in the women. Our study initially requested information from the women about their family history. In our experience, most women are aware of the inherited predisposition to breast cancer and, as a result, are unlikely to be distressed by the request of family history information regarding cancer. The subsequent steps were guided by the women who were at liberty to decline further contact. We accept that further work is required to assess the effects fully (psychological sequelae, risk perception, modification of behaviour, and the likelihood and repercussions of information dissemination within the family) of offering genetic counselling to the women in this study. Evans et al, ${ }^{14}$ in a study to identify the risk perceptions of women attending a cancer genetic clinic, found that only $11 \%$ estimated their risk accurately and $41 \%$ underestimated their risk. They suggested that the women who underestimated their risk were likely to be worried by assessments of higher risk. In contrast, Lerman et $a l^{15}$ in a randomised, prospective trial of individualised breast cancer risk counselling versus general health counselling, offered to a cohort of women with one or more first degree relatives affected with breast cancer, found that the women offered specific breast cancer risk information were more likely to estimate their risk correctly. Their work also indicated that, in both groups, around two thirds of the women continued to overestimate their lifetime risk substantially, in spite of counselling. They raise the concern that the exaggerated risk perception in these women may reduce adherence to mammography guidelines, ${ }^{16}$ lead to avoidance of breast self examination, ${ }^{17}$ and impact adversely on quality of life. ${ }^{18}$ They also raise concerns about the risk of inappropriate decision making by women (for example, request for prophylactic mastectomy) who falsely perceive their risks to be increased. These authors found that the women with increased levels of anxiety are least likely to improve their personal risk estimation, and their work indicates the need for further research in this area. We accept that our intervention may have caused similar worries to the subjects in this study and investigations are under way to assess this further. Many women seen in the present study have considered their family history to confer an increased risk to themselves and welcomed the opportunity to discuss this.

Among the high penetrance breast cancer predisposing genes that have been identified is the BRCA1 gene on chromosome $17 \mathrm{q}$, implicated in breast and epithelial ovarian cancer and a proportion of early onset breast cancer families. ${ }^{19}$ Another gene, termed BRCA2, has now been linked to a locus on chromosome $13,{ }^{20}$ and this has been implicated in families with male and female breast cancers. Four women in the study have developed cancer (discussed in Results). In two cases, both the presentation and their family history are strongly suggestive of BRCA1 linked cancer predisposition. ${ }^{19}$ The third person's family history is suggestive of hereditary non-polyposis colorectal cancer (HNPCC), ${ }^{21} 22$ a dominantly inherited predisposition to colorectal and other cancers. The fourth person may represent a sporadic breast cancer in spite of her family history. Molecular studies are under way to investigate these families further for evidence 
of germline mutations in the BRCA1 gene ${ }^{19}$ in the case of the breast and ovarian cancer families and the hMSH $2^{2122}$ gene in the HNPCC family. None of the affected women expressed any regret at participating in this work and were pleased that other family members would be benefiting from continuing research.

Previous studies to identify the family history in women attending for screenings have been flawed by the screening sample being self selected and the lack of strict selection criteria for a family history likely to confer an increased risk to the women. ${ }^{2324}$ Our findings suggest that around $1 \cdot 24 \%$ of women attending the NHSBSP (including 154 of the consultands and 289 of their relatives between 50 and 64) have a family history of breast cancer conferring an increased risk to themselves. This is likely to be an underestimate as some women may not have divulged family history information, the radiographers may not have requested information, and the radiologists may have failed to recall the women in some instances. A further $0.26 \%$ have a family history conferring an increased risk to other family members. We have shown that these women and their at risk relatives can be effectively identified and targeted for appropriate counselling and more frequent mammography. In spite of the process being labour intensive, we feel that women identified in this manner should also be offered genetic counselling to evaluate risk accurately and target appropriate screening to those people who are likely to benefit from more frequent mammography or wish to pursue the options for preventive therapy. A few may also be offered information about the availability of genetic testing for themselves and other family members. The work of Lerman et $a l^{25}$ indicates that subjects who attended their health promotion trial had higher levels of anxiety and education than those who declined. Offering these women at increased risk of breast cancer screening only, without access to genetic counselling, may be potentially more detrimental, in reinforcing perceived risks and provoking more anxiety. An important aim of the counselling is to help the women to make informed choices about the management options available to them. Making informed choices about screening and other options is particularly important for the at risk female relatives under 50 , in whom mammography has not been proven to be of benefit in reducing breast cancer mortality (although a meta analysis of the Swedish trial ${ }^{26}$ of breast screening showed a $13 \%$ non-significant benefit in the 40 to 50 year age group). A cancer genetic clinic may be the best place to offer such a service, although we accept that the true costs (economic, psychological) and benefits (reduced breast cancer mortality and morbidity, increased awareness among the at risk women and their doctors, informed decision making on treatment choices by the at risk women) of such genetic counselling remain to be proven.

D de Silva was funded by the Aberdeen Royal Hospitals NHS Trust. The authors thank colleagues in the North East Scotland Breast Screening Centre and the Medical Genetics Department for their help. We also thank the families who participated in this work and their general practitioners, surgeons, and gynaecologists.

1 Forrest P. Breast cancer screening: report to the health ministers of England, Wales, Scotland and Northern Ireland. London: of England, Wales,

2 Registrar General for Scotland annual report 1992. London: Registrar General

3 Go R. Genetic epidemiology of breast cancer and associated cancers in high risk families. 1 . Segregation analysis. $\mathcal{F}$ Natl Cancer Inst 1983;71:455-61.

4 Williams W, Anderson D. Genetic epidemiology of breast cancer: segregation analysis of 200 Danish pedigrees. Genet Epidemiol 1984;1:7-20.

5 Newman B, Austin M, Lee M, King M. Inheritance of human breast cancer: evidence for autosomal dominant transmission in high risk families. Proc Natl Acad Sci USA 1988;85:3044-8.

6 Claus E, Risch N, Thompson W. Age at onset as an indicator of familial risk of breast cancer. Am $\mathcal{F}$ Epidemiol 1990;131: 961-72.

7 Houlston R, McCarter E, Parbhoo S, et al. Family history and risk of breast cancer. $\mathcal{f}$ Med Genet 1992;29:154-7.

8 Mettlin C, Croghan I, Natarajan N, et al. The association of age and familial risk in a case control study of breast of age and familial risk in a case control

Margaritte P, Bonaiti-Pellie C, King MC, et al. Linkage of familial breast cancer to chromosome $17 \mathrm{q} 21$ may not be familial breast cancer to chromosome $17 \mathrm{q} 21$ may not be restricted to
$\mathbf{5 0 : 1 2 3 1 - 4}$

10 Merette C, Ott J. Is there heterogeneity of age of onset for breast cancer? Am $\mathcal{F}$ Hum Genet 1993;52:627-9.

11 Ford D, Easton D, Bishop D, et al. Risks of cancer in BRCA1 mutation carriers. Lancet 1994;343:692-5.

2 Claus E, Risch N, Thompson D. Genetic analysis of breas cancer in the cancer and steroid hormone study. $A m \mathcal{J}$ Hum Genet 1991;48:232-42.

13 Rudiman R, Gilbert F, Ritchie L. Comparison of uptake of breast screening, cervical screening and childhood immunisation. BMf 1995;310:229.

14 Evans D, Burnell L, Hopwood P, et al. Perception of risk in women with a family history of breast cancer. $\mathrm{Br} \mathcal{F}$ Cancer 1993;67:612-14.

15 Lerman C, Lustbader E, Rimer B, et al. Effects of individualised breast cancer risk counselling: a randomized dividualised breast cancer risk counsellin

16 Lerman C, Daly M, Sands C, et al. Mammography adherence and psychological distress among women at ris of breast cancer. $\mathcal{F}$ Natl Cancer Inst 1993;85:1074-80.

17 Kash K, Holland J, Stefanek M, et al. Psychological distres and surveillance behaviour of women with a family history of breast cancer. $\mathcal{F}$ Natl Cancer Inst 1992;84:24-30.

18 Lerman C, Schwartz M. Adherence and psychological adjustment among women at high risk for breast cancer Breast Cancer Res Treat 1993;28:145-55.

19 Miki Y, Swenson J, Schattuck-Eidens D, et al. A strong candidate for the breast and ovarian cancer susceptibility gene BRCA1. Science 1994;266:66-71.

20 Wooster R, Neuhausen S, Manglon J, et al. Localization of a breast cancer susceptibility gene BRCA2 to chromosome 13q12-13. Science 1994;265:2088-90.

21 13q12-13. Science 1994;265:2088-90. , of the mutS homolog in heredit.

22 Papadopoulos N, Nicolaides N, Wei Y, et al. Mutations in Papadopoulos N, Nicolaides N, Wei Y, et al. Mutations in 1994;263:1625-9.

23 Polednak A, Lane D, Burg M. Risk perception, family history and use of breast cancer screening tests. Cancer Detect Prevent 1991;15:257-63.

24 Bondy M, Vogel V, Halabi S, et al. Identification of women at increased risk of breast cancer in the population based screening programme. Cancer Epidemiol, Biomarkers Prevent $1992 ; 1: 143-7$

25 Lerman C, Rimer B, Daly M, et al. Recruiting high risk women into a breast cancer health promotion trial. Cancer Epidemiol Biomarkers Prevent 1994;3:271-6.

26 Chamberlain J. Firmer evidence on the value of breast screening - the Swedish overview. Eur f Cancer 1993;29: 1804-5. 